\title{
Flumorph Is a Novel Fungicide That Disrupts Microfilament Organization in Phytophthora melonis
}

\author{
Shu Sheng Zhu, Xi Li Liu, Peng Fei Liu, Yong Li, Jian Qiang Li, Hui Min Wang, Shan Kui Yuan, and Nai Guo Si
}

\begin{abstract}
First, second, third, fifth, and sixth authors: Department of Plant Pathology, China Agricultural University, Beijing 100094, China; fourth author: Department of Human Nutrition, Food and Animal Sciences, University of Hawaii; seventh author: Center of Agrochemicals for Biological and Environmental Technology, Institute for the Control of Agrochemicals, Ministry of Agriculture, Beijing 100026, China; and eighth author: China Shenyang Research Institute of Chemical Industry, Shenyang 110021, China.
\end{abstract}

Accepted for publication 27 October 2006.

\begin{abstract}
Zhu, S. S., Liu, X. L., Liu, P. F., Li, Y., Li, J. Q., Wang, H. M., Yuan, S. K., and $\mathrm{Si}$, N. G. 2007. Flumorph is a novel fungicide that disrupts microfilament organization in Phytophthora melonis. Phytopathology 97:643-649.

The mechanism of the effects of flumorph (a novel fungicide) was investigated by analyzing alterations of hyphal morphology, cell wall deposition patterns, F-actin organization, and other organelles in Phytophthora melonis. Calcofluor white staining suggested that flumorph did not inhibit the synthesis of cell wall materials, but disturbed the polar deposition of newly synthesized cell wall materials during cystospore germination and hyphal growth. After exposure to flumorph, zoospores were able to switch into cystospores accompanied with the formation of a cell wall, whereas cystospores failed to induce the isotropic-polar switch and did not produce germ tubes but continued the isotropic growth phase.

tion of tip growth. Newly synthesized cell wall materials were deposited uniformly throughout the diffuse expanded region of hyphae, in contrast to their normal polarized patterns of deposition. These alterations were the result of F-actin disruption, identified with the fluorescein isothiocynate (FITC)-phalloidin staining. The disruption of F-actin also was accompanied by disorganized organelles: each swelling of subapical hyphae was associated with a nucleus. Vesicles did not undergo polarized secretion to the apical hyphae, but diffused around nuclei for the subapical growth; thus, the cell wall was thickened with periodic expansion along the hyphae. Upon removing flumorph, normal tip growth and organized F-actin were observed again. These data, as well as data published earlier, suggest that flumorph may be involved in the impairment of cell polar growth through directly or indirectly disrupting the organization of Factin. The primary site of action by flumorph in the disruption of the Factin organization is under investigation.
\end{abstract} In flumorph-treated hyphae, the most characteristic change was the development of periodic swelling ("beaded" morphology) and the disrup-

Flumorph, 4-[3-(3,4-dimethoxyphenyl)-3-(4-fluorophenyl)-1oxo-2-propenyl] morpholine, a derivative of cinnamic acid, is a new systemic fungicide developed for the control of oomycete pathogens by Shenyang Research Institute of Chemical Industry of China in 1994 (20,32). It exhibits a very high level of activity against members of the family Peronosporaceae and the genus Phytophthora but not Pythium. Flumorph has been patented in China (ZL.96115551.5), the United States (US6020332), and Europe (0860438B1) and is registered for controlling Phytophthora infestans on potato and tomato, $P$. capsici on pepper, Pseudoperonospora cubensis on cucumber, and Plasmopara viticola on grapevine in China (21).

Flumorph inhibits all stages in the life cycle of Pseudoperonospora cubensis except for zoospore discharge and motility and cystospore formation (33). This inhibition is identical to that of dimethomorph, but different from other commercial fungicides $(2,6,33)$. In addition, flumorph and dimethomorph have similar compound structures $(21,31)$ and identical pathogen-resistance patterns (31). Thus, flumorph and dimethomorph could have novel biochemical targets and exhibit the same (or similar) mechanisms for controlling diseases. However, no data are available regarding the biochemical target of flumorph. Previously published data indicated that dimethomorph may interfere with the assembly of wall polymers in the fungal cells, but its primary

Corresponding author: X. L. Liu; E-mail address: seedling@ cau.edu.cn

doi:10.1094/PHYTO-97-5-0643

(c) 2007 The American Phytopathological Society
Additional keywords: mode of action.

mode of action also remains inconclusive (19). Thus, it is very important to elucidate the fungicidal mechanism of flumorph and dimethomorph for development of new fungicides and fungicide resistance management.

In this study, we employed a staining method using calcofluor white to observe the synthesis and assembly of the cell wall during cystospore germination and hyphal growth of Phytophthora melonis Katsura after exposure to flumorph. We also explored the effect of flumorph on F-actin distribution and the consequences of F-actin disruption in hyphae, which allowed us to more clearly analyze the mode of action of flumorph.

\section{MATERIALS AND METHODS}

Origin and maintenance of fungi. $P$. melonis isolates TJ-58 from China (sensitive to flumorph) and FR-58 (resistant to flumorph) were used in this study. FR-58 was selected from TJ-58 after mass screening of sporangia on solid white kidney bean agar (WKB) medium containing flumorph at $20 \mathrm{mg} / \mathrm{liter}$, a concentration at which the growth of TJ-58 is completely inhibited. Cultures were maintained routinely on WKB agar at $25^{\circ} \mathrm{C}$ in darkness and new subcultures were made weekly.

Growth inhibition assays. The sensitivity of hyphal growth of TJ-58 and FR-58 to flumorph was determined according to a previous method (24). Mycelial plugs, $5 \mathrm{~mm}$ in diameter, were cut from the growing edge of a colony maintained on WKB plate and placed on a series of WKB plates containing flumorph at different concentrations $(0,0.01,0.05,0.07,0.1,0.15,0.2,0.25,0.3,0.4$, $0.5,1,5,10,15,20$, and $25 \mathrm{mg} / \mathrm{liter}$ ). In all cases, the final 
amount of solvent never exceeded $1 \%(\mathrm{vol} / \mathrm{vol})$ in treated and control samples. Growth was assessed by measuring the increase in colony diameter after incubation at $25^{\circ} \mathrm{C}$ for 4 days in darkness, and $50 \%$ effective concentration $\left(\mathrm{EC}_{50}\right)$ values for growth inhibition were determined from dose-response curves.

The sensitivity of cystospore germination of TJ-58 and FR-58 to flumorph was measured as described previously for dimethomorph $(2,24)$, with a few modifications. An aliquot of $20 \mu \mathrm{l}$ of suspension containing $1 \times 10^{4}$ zoospores $/ \mathrm{ml}$ was transferred to depression glass slides. Then, $20 \mu \mathrm{l}$ of a flumorph solution of various concentrations $(0,0.02,0.1,0.14,0.2,0.3,0.4,0.5,0.6$, $0.8,1,2,10,20,30,40$, and $50 \mathrm{mg} /$ liter) was added immediately

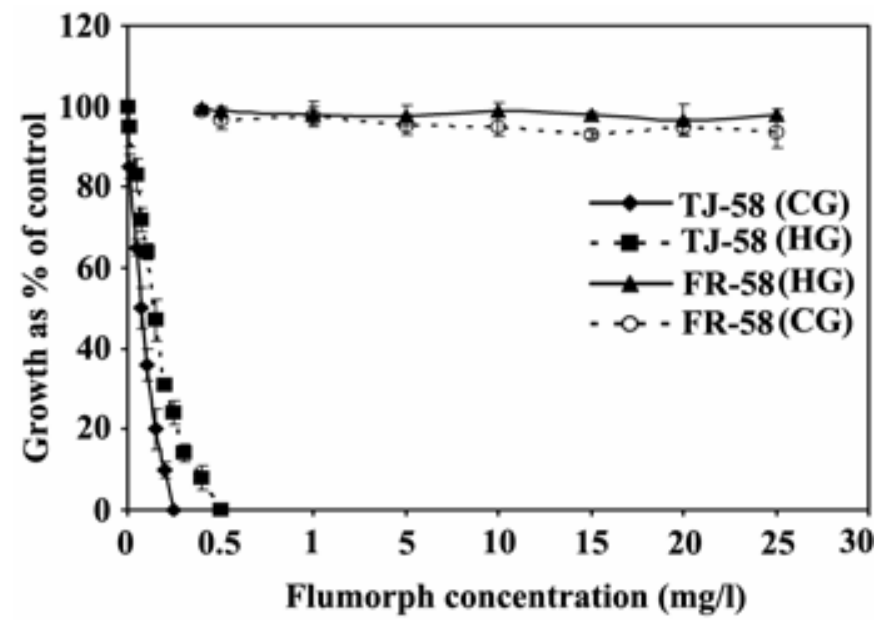

Fig. 1. Sensitivity of cystospore germination (CG) and hyphal growth (HG) of wild-type isolate TJ-58 and flumorph-resistant isolate FR-58 of Phytophthora melonis to flumorph. Results are means of three replications with bars showing the standard errors. to the suspension, and the rate of germination was visualized under a microscope after incubation in darkness at $19^{\circ} \mathrm{C}$ for $4 \mathrm{~h}$. $\mathrm{EC}_{50}$ values for germination inhibition of cystospores were determined from dose-response curves.

Synthesis and deposition patterns of cell wall materials during cystospore formation and germination. An aliquot of $20 \mu \mathrm{l}$ of suspension containing $1 \times 10^{4}$ zoospores/ml was transferred to depression glass slides. A drop $(20 \mu \mathrm{l})$ of flumorph solution at concentrations of $0.5,1,5,10$, and $50 \mathrm{mg} / \mathrm{liter}$ was added immediately to each slide containing the zoospore suspension. Subsequently, new synthesis of cell wall materials when cystospores formed and the deposition patterns of cell wall materials during cystospore germination were observed under a fluorescent microscope after staining with calcofluor white $(0.01 \%$, wt $/ \mathrm{vol})$.

Effect of flumorph on the morphology and cell wall formation of hyphae. The effect of flumorph on hyphal morphology was visualized as described previously (19). Briefly, WKB agar was overlaid with a cellophane membrane (Bio-Rad Laboratories, Inc.), inoculated with a mycelial plug, and incubated for 3 days at $25^{\circ} \mathrm{C}$ in darkness. Under these conditions, a relatively sparse hyphal monolayer was produced which could be observed directly under the light microscope, or the uppermost undisturbed layer could be removed intact on the cellophane membrane after exposure to the fungicide. Cellophane cultures with mycelium on top were submerged for $5 \mathrm{~min}$ in filtered WKB broth containing flumorph at a concentration of $25 \mathrm{mg} / \mathrm{liter}$. Excess liquid was absorbed and the cellophane cultures were incubated for $8 \mathrm{~h}$ before examination of the morphological changes of hyphae with a compound microscope (Olympus CH-2; Japan). Subsequently, the synthesis and deposition patterns of new cell wall materials in flumorph-treated hyphae were observed under a fluorescent microscope after staining with $0.01 \%$ calcofluor white (wt/vol).

F-actin distribution. The effect of flumorph on F-actin disruption was investigated by incubating hyphae in WKB broth amended with flumorph at concentrations of 1,5 , and $25 \mathrm{mg} / \mathrm{liter}$

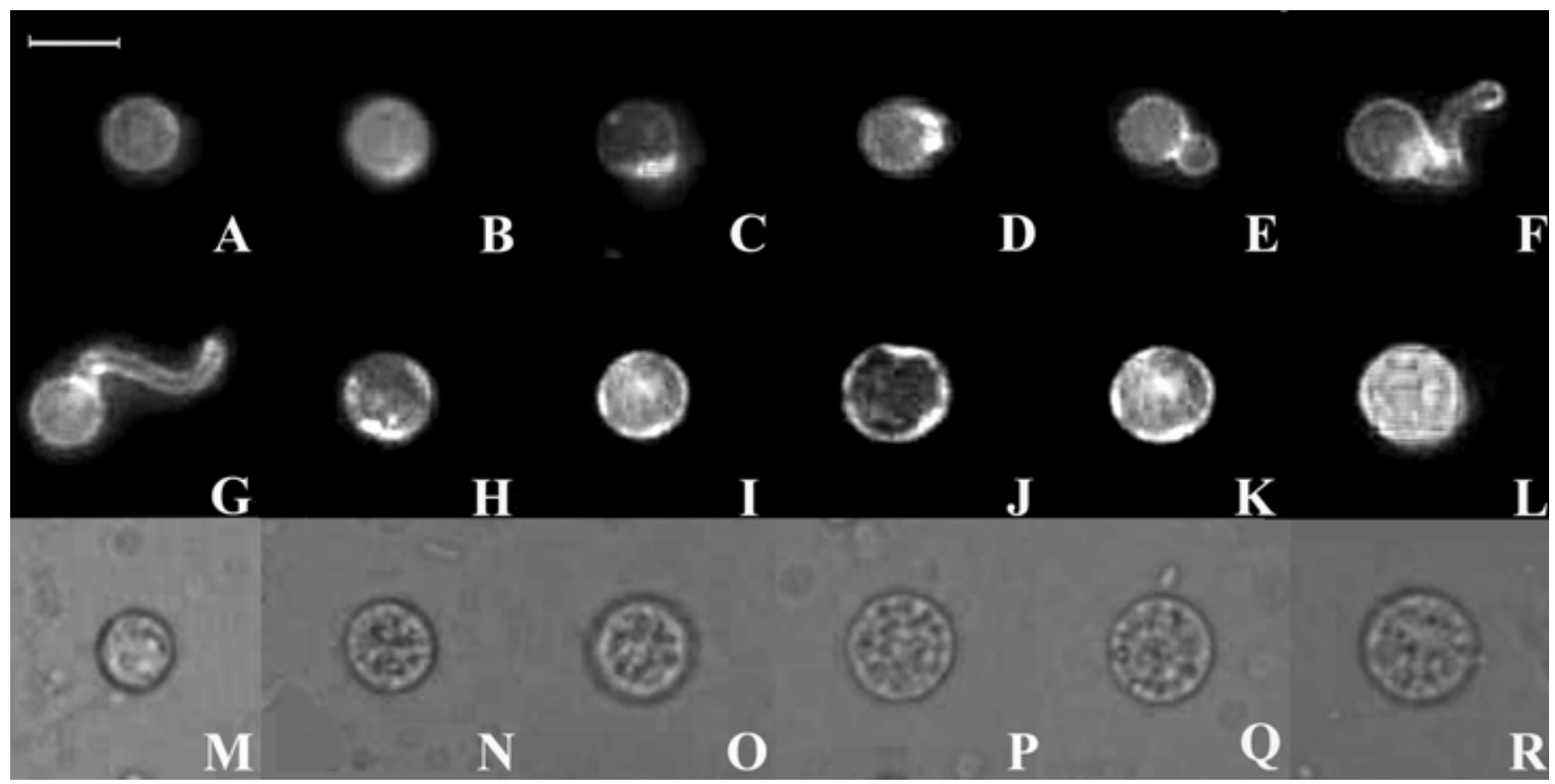

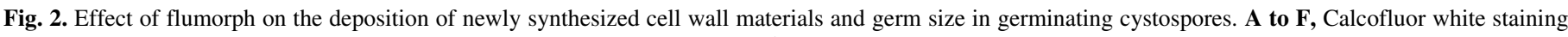

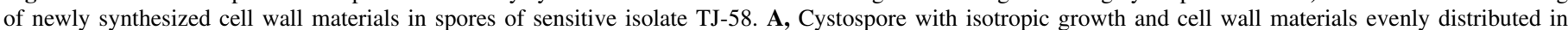

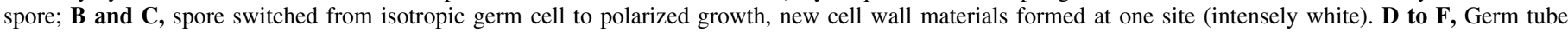

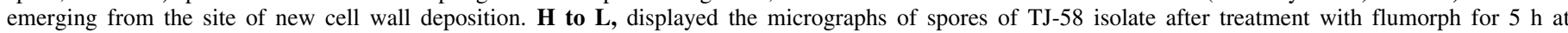

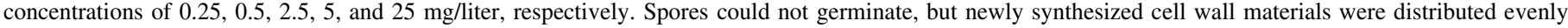

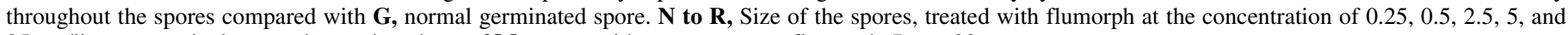
$25 \mathrm{mg} /$ liter, respectively, were larger than those of $\mathbf{M}$, spores without exposure to flumorph. Bar $=20 \mu \mathrm{m}$. 
for 2, 4, and $8 \mathrm{~h}$, and then visualizing F-actin organization as described previously (26). Briefly, visualization of the actin cytoskeleton was done using fluorescein isothiocynate (FITC)phalloidin. Hyphae were rinsed in phosphate-buffered saline (PBS) alone, fixed in $3.7 \%$ formaldehyde for $30 \mathrm{~min}$, washed twice with PBS containing 0.03\% Triton X-100 (PBST), and resuspended in $100 \mu \mathrm{l}$ of PBST. A solution $(10 \mu \mathrm{l})$ of $6.6 \mu \mathrm{M}$ in $\mathrm{MeOH}$ FITC-phalloidin (Molecular Probes, Eugene, OR) was added and the hyphae were incubated for $30 \mathrm{~min}$ in darkness. Subsequently, hyphae were washed five times in PBST and resuspended in $50 \mu \mathrm{l}$ of mounting medium $(50 \mathrm{mg}$ of $\mathrm{p}$ phenylenediamine in $5 \mathrm{ml}$ of PBS, pH 8.0 [adjusted with $0.5 \mathrm{M}$ $\mathrm{Na}_{2} \mathrm{CO}_{3} ; \mathrm{pH}$ 9.0] in $50 \mathrm{ml}$ with glycerol). F-actin organization was visualized with a confocal microscope (Leica Lasertechnik GmbH, Heidelberg, Germany).

Recovered growth of hyphal tip. To determine whether the effect of flumorph was reversible, the hyphae, which were grown on cellophane placed on top of the growth medium, were immersed in WKB broth amended with flumorph at $25 \mathrm{mg} / \mathrm{liter}$ for $3 \mathrm{~h}$, and the flumorph subsequently was removed by rinsing three times with washes of WKB broth. The hyphae were incu- bated at $2^{\circ} \mathrm{C}$ for up to $21 \mathrm{~h}$, during which the diameter of colonies was measured after $3,7,10,14$, and $21 \mathrm{~h}$. Other evaluations included growing the hyphae in WKB broth without flumorph or in the continued presence of flumorph at $25 \mathrm{mg} / \mathrm{liter}$. The changes of hyphal morphology, F-actin organization, and cell wall synthesis after flumorph washout were observed according to the above methods.

Ultrastructure of hyphae. The extreme cytological effects of treating hyphae with flumorph at $25 \mathrm{mg} / \mathrm{liter}$ for 8,16 , and $24 \mathrm{~h}$ were determined with the use of a transmission electron microscope (TEM), utilizing conventional fixation (19). Briefly, "target" areas of the colony, grown on a cellophane membrane, were located by light microscopy and removed from sections of the membrane without disturbance of the mycelium and fixed in situ. Specimens were subjected to primary fixation for $2 \mathrm{~h}$ in $3 \%$

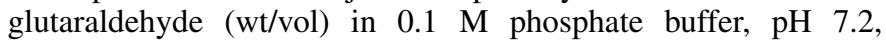
followed by several rinses in the same buffer, totaling $10 \mathrm{~min}$. Post-fixation was in 1\% OsO4 (wt/vol) in the above buffer for $1 \mathrm{~h}$ followed by repeated rinses in distilled water, totaling $20 \mathrm{~min}$. Block staining was done in $2 \%$ aqueous uranyl acetate (wt/vol) for $1 \mathrm{~h}$ in darkness followed by dehydration in an acetone series

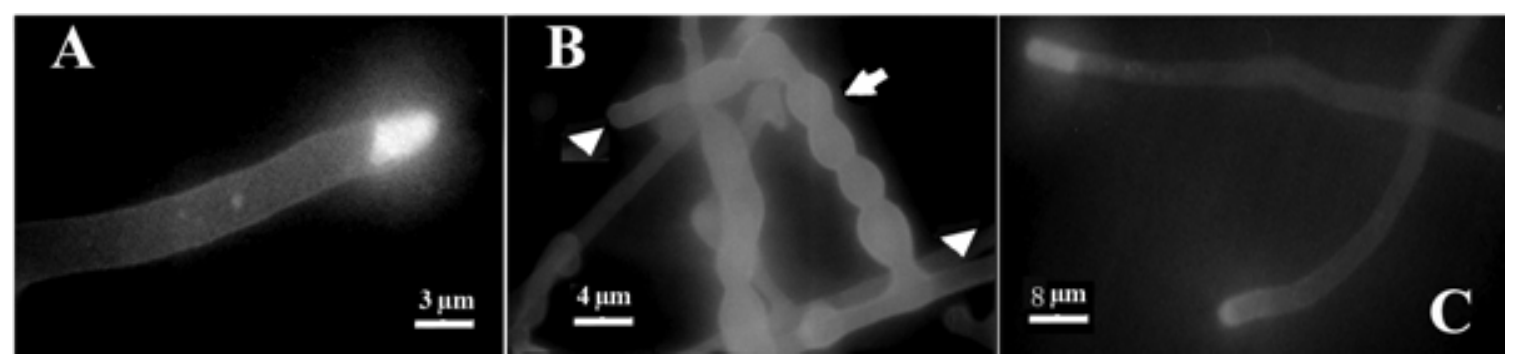

Fig. 3. Morphology and deposition of newly synthesized cell wall materials in the hyphae of sensitive isolate TJ-58 and resistant isolate FR-58 of Phytophthora melonis after exposure to flumorph. A, After calcofluor white staining, the cell walls of new, normal tips of TJ-58 were stained intensely. B, However, only regions of diffuse expansions stained intensely (arrow) in the hyphae of TJ-58 after treatment with flumorph at $25 \mathrm{mg} /$ liter compared with the hyphal tip (arrowhead) and older, nonexpanding walls (arrowhead). C, Tips of FR-58 still were stained intensely after exposure to flumorph.

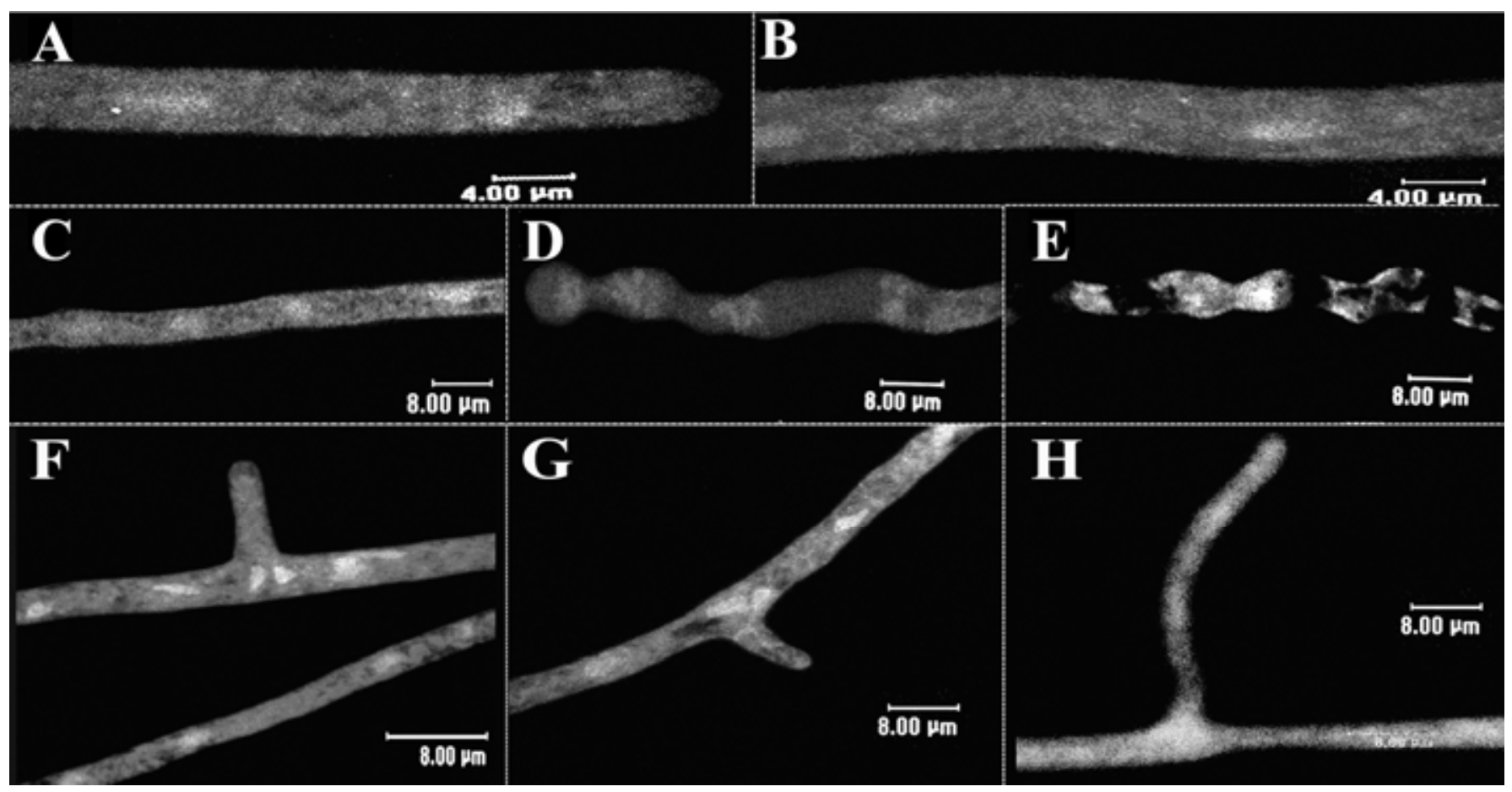

Fig. 4. Organization of F-actin in hyphae of Phytophthora melonis of wild-type (TJ-58) and flumorph-resistant (FR-58) isolates after exposure to flumorph. Factin in tips and subapical regions of hyphae of the wild type exposed to flumorph at $\mathbf{C}, 1, \mathbf{D}, 5$, and $\mathbf{E}, 25 \mathrm{mg} /$ liter for $8 \mathrm{~h}$ became increasingly disrupted in comparison to $\mathbf{A}$ and $\mathbf{B}$, the normal organization. However, the organization of F-actin in the hyphae of FR-58 isolate was not disrupted after exposure to flumorph at $25.0 \mathrm{mg} / \mathrm{liter}$ for $\mathbf{F}, 2, \mathbf{G}, 4$, and $\mathbf{H}, 8 \mathrm{~h}$. 
and infiltration in Spurr resin, and stained with lead citrate before examination at 60 to $80 \mathrm{kV}$ in a Jeol $100 \mathrm{~S}$ electron microscope.

Nuclear staining. In order to determine whether disruption of flumorph-induced F-actin also altered other cell components, nuclei were stained with 9,6-diamidino-2-phenylindole dihydrochloride (DAPI) (Sigma-Aldrich) at $10 \mathrm{mg} / \mathrm{liter}$, an intercalating dye of DNA (29). The samples were treated as described for Factin staining and observed with a fluorescent microscope.

\section{RESULTS}

Fungicidal effects of flumorph on cystospore germination and hyphal growth. Flumorph had almost no effect on spore germination and hyphal growth of resistant isolate (FR-58) at concentrations of up to $25 \mathrm{mg} / \mathrm{liter}$. However, concentrations of 0.25 and $0.5 \mathrm{mg} /$ liter completely inhibited cystospore germination and hyphal growth, respectively, of sensitive isolate TJ-58 (Fig. 1).

Effect on the synthesis and deposit patterns of cell wall materials during cystospore formation and germination. The synthesis and distribution of cell wall materials during cystospore formation and germination were observed with a fluorescence microscope after staining with calcofluor white, a dye with high affinity for $\beta$-polysaccharides, such as cellulose and chitin (14). Calcofluor white intensely stains the newly synthesized apical cell wall materials but does not react strongly with older, subapical regions. Thus, it often is used to show the synthesis and deposition of newly synthesized cellulose or chitin in cells $(4,27)$. Be-

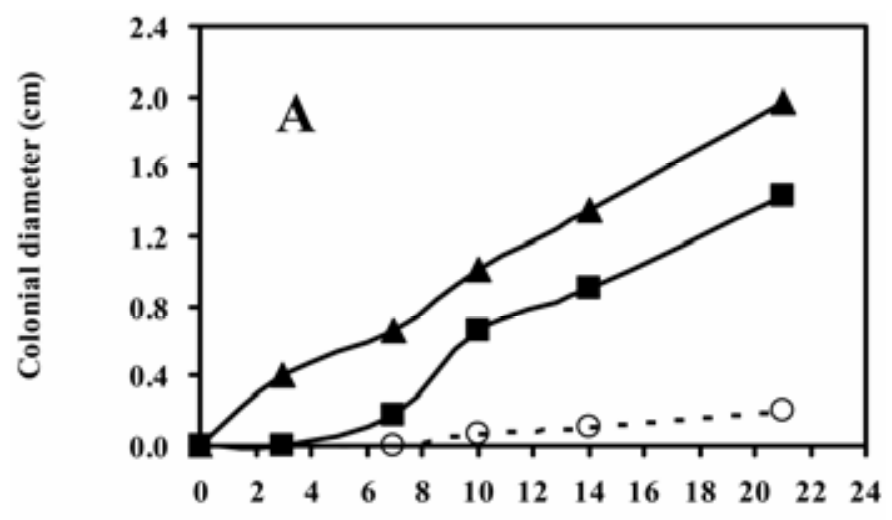

Time (h)

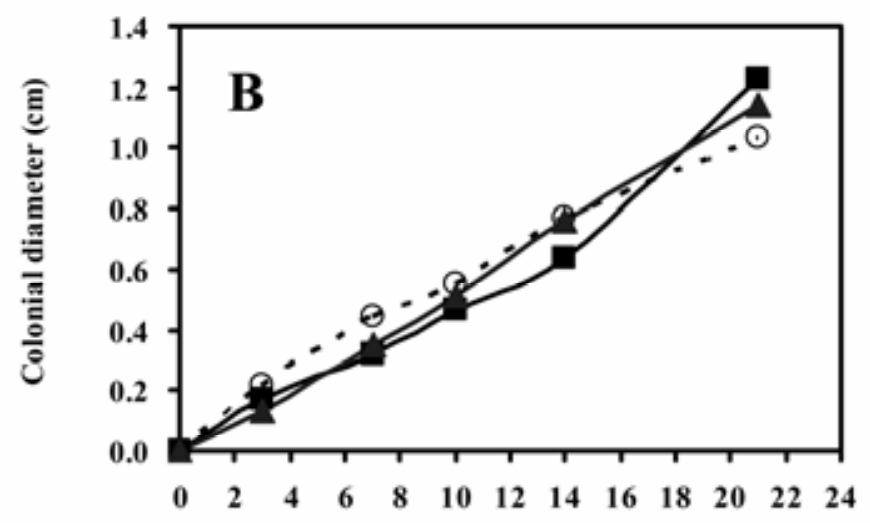

Time (h)

Fig. 5. Effects of flumorph on colonial growth of wild-type isolate TJ-58 and flumorph-resistant isolate of Phytophthora melonis. A and B, Dynamic growth of colony diameter of TJ-58 and FR-58 after treatment with flumorphfree white kidney bean $(\mathrm{WKB})$ broth $(\boldsymbol{\Delta})$, flumorph at $25.0 \mathrm{mg} /$ liter $(\mathrm{O})$, or flumorph at $25.0 \mathrm{mg} /$ liter for $3 \mathrm{~h}$ and then washed out with flumorph-free WKB broth ( $\bullet$ ). cause zoospores and the initial stage of cystospores are free from the cell wall, they cannot be stained by calcofluor white. If flumorph inhibits the synthesis of cell wall materials, zoospores will not be transformed into cystospores and will not be stained with calcofluor white. In our tests, strong calcofluor fluorescence was seen in cystospores whether they were treated (Fig. $2 \mathrm{H}$ to $\mathrm{L}$ ) or untreated (Fig. 2A) with flumorph, indicating the synthesis of cell wall materials. However, the distribution of newly synthesized cell wall materials was disturbed during cystospore germination. Germination of control cystospores was initiated by an isotropic growth phase to generate spherical cells (Fig. 2A). After an incubation period of 0.5 to $1 \mathrm{~h}$, the cystospores began to switch from isotropic to polar growth, with uniform distribution of cell wall materials throughout the cell converging to a central point (Fig. 2B and C), where the hyphal tube emerged (Fig. 2D and E). At the tip of the germ tube and the site of germ tube emergence, the cell wall was strongly stained (Fig. 2F). This showed that the newly synthesized cell wall materials were concentrated. However, after treatment with flumorph at concentrations of $0.25,0.5$, $2.5,5$, and $25 \mathrm{mg} / \mathrm{liter}$, cystospores failed to induce the isotropicpolar switch and, thus, did not produce germ tubes. However, cytospores continued the isotropic growth phase, which was marked by uniform distribution of newly synthesized cell wall materials throughout the cell (Fig. $2 \mathrm{H}$ to L). This isotropic growth phase continued beyond the flumorph-free cystospores size, resulting in double-sized cystospores (Fig. 2M to R). For the resistant isolate, however, cystospore germination and germ size were not affected by flumorph.

Flumorph induced changes in hyphal morphology and cell wall deposition. Flumorph at a concentration of $25 \mathrm{mg} / \mathrm{liter}$ completely inhibited the colony growth of sensitive isolates (Fig. 1). It also induced major changes in the morphology of hyphae of sensitive isolates, and the most characteristic effect of flumorph was the development of periodic swellings along the hyphae, producing a "beaded" appearance (Fig. 3B). However, the morphology of the hyphae of the resistant isolate was not significantly affected by flumorph, and they grew normally (Fig. 3C).

The altered hyphae were stained with calcofluor white to view the distribution of newly synthesized cell wall materials using fluorescent microscopy. The cell walls of new, normal tips were stained intensely (Fig. 3A), an indication of new wall synthesis. After treatment with flumorph at $25 \mathrm{mg} / \mathrm{liter}$, only the regions of swellings were stained with intense fluorescence, compared with hyphal tip and older, nonexpanding walls (Fig. 3B), indicating that they were regions of new growth with wall properties similar to newly synthesized walls. However, the hyphal tips of resistant isolate FR-58 still were stained intensely after treatment with flumorph at $25 \mathrm{mg} /$ liter (Fig. 3C).

Flumorph induces F-actin disruption in hyphae. Previous studies showed that the site of cell wall deposition coincides with the location of the F-actin $(22,27)$ and the polar growth of fungi are dependent on the integrity of the actin cytoskeleton $(11,15)$. Thus, we explored the effect of flumorph on F-actin organization. In normal hyphae, the F-actin was distributed evenly in apical (Fig. 4A) and subapical (Fig. 4B) hyphae. However, the organization of F-actin was disrupted by flumorph after $8 \mathrm{~h}$ at all concentrations of the drug. The F-actin was slightly disrupted by flumorph at $1 \mathrm{mg} / \mathrm{liter}$ (Fig. 4C) and concentrated along the hyphae. It was further disrupted by flumorph at concentrations of 5 and $25 \mathrm{mg} / \mathrm{liter}$, and the swellings of hyphae had most of the concentrated F-actin (Fig. 4D and E). However, F-actin was not disrupted in the hyphae of a flumorph-resistant isolate after exposure to flumorph at $25 \mathrm{mg} /$ liter for $2 \mathrm{~h}$ (Fig. 4F), $4 \mathrm{~h}$ (Fig. $4 \mathrm{G})$, and $8 \mathrm{~h}$ (Fig. 4H).

Recovered growth of hyphal tip after flumorph washout. Mycelial growth of the wild isolate TJ-58 was significantly slowed after the addition of flumorph at a concentration of $25 \mathrm{mg} /$ liter. After washing off the drug, however, growth inhi- 
bition induced by flumorph at $25 \mathrm{mg} /$ liter for $3 \mathrm{~h}$ was reversible and the rate of growth was similar to that of the untreated hyphae (Fig. 5A). However, colony diameter of resistant isolates was not affected at all, whether treated with flumorph or not (Fig. 5B). After observation with a microscope, we visualized that new normal, tubular hyphae emerged from the bulge of hyphal tip of sensitive isolates again and that F-actin also reappeared in the hyphal tip (data not shown).

Effect of flumorph on hyphal ultrastructure. Concomitant with the disruption of F-actin, there were ultrastructural changes in hyphae. Control mycelia of $P$. melonis, grown in the absence of flumorph, showed many of the cytological and ultrastrustural features typical of vegetative hyphae of the genus (Fig. 6A and $\mathrm{G})$. The most pronounced ultrastructural change observed in hyphae after exposure to flumorph was the extensive cell wall thickness. Wall thickness increased throughout the colony in the continued presence of flumorph for $8 \mathrm{~h}$ from the normal $0.3 \mu \mathrm{m}$ (Fig. 6A) to $\approx 1.5 \mu \mathrm{m}$ (Fig. 6B and C). Hyphae incubated with flu- morph for $24 \mathrm{~h}$ showed multiple layers of cell walls containing entrapped cytoplasmic elements (Fig. 6C and D). The flumorph also induced alterations in organelle distributions. The mitochondria (Fig. 6E) and nuclei (Fig. 6F) apparently were unaffected, and vacuoles (Fig. 6F) were found throughout the cytoplasm. Also, the vesicles apparently lost their normal polarized concentration in the apical portion of hyphae (Fig. 6G), because they frequently were found in the subapical regions, often adjacent to the thick region of wall (Fig. $6 \mathrm{H}$ ) and the multiple layers (Fig. 6I).

Effect of flumorph on nuclei distribution in hyphae. After staining with DAPI, we observed that nuclei were evenly distributed in the normal hyphae; the average distance between adjacent nuclei in several hyphae from a typical colony was $8.98 \pm 1.73 \mu \mathrm{m}(n=21)$ (Fig. 7A). The average distance between adjacent nuclei in the flumorph-treated hyphae of sensitive and resistant isolates was $9.29 \pm 1.12 \mu \mathrm{m}(n=18)$ and $9.08 \pm 1.42$ $(n=23)$, respectively (Fig. 7B and C). They were still similar to

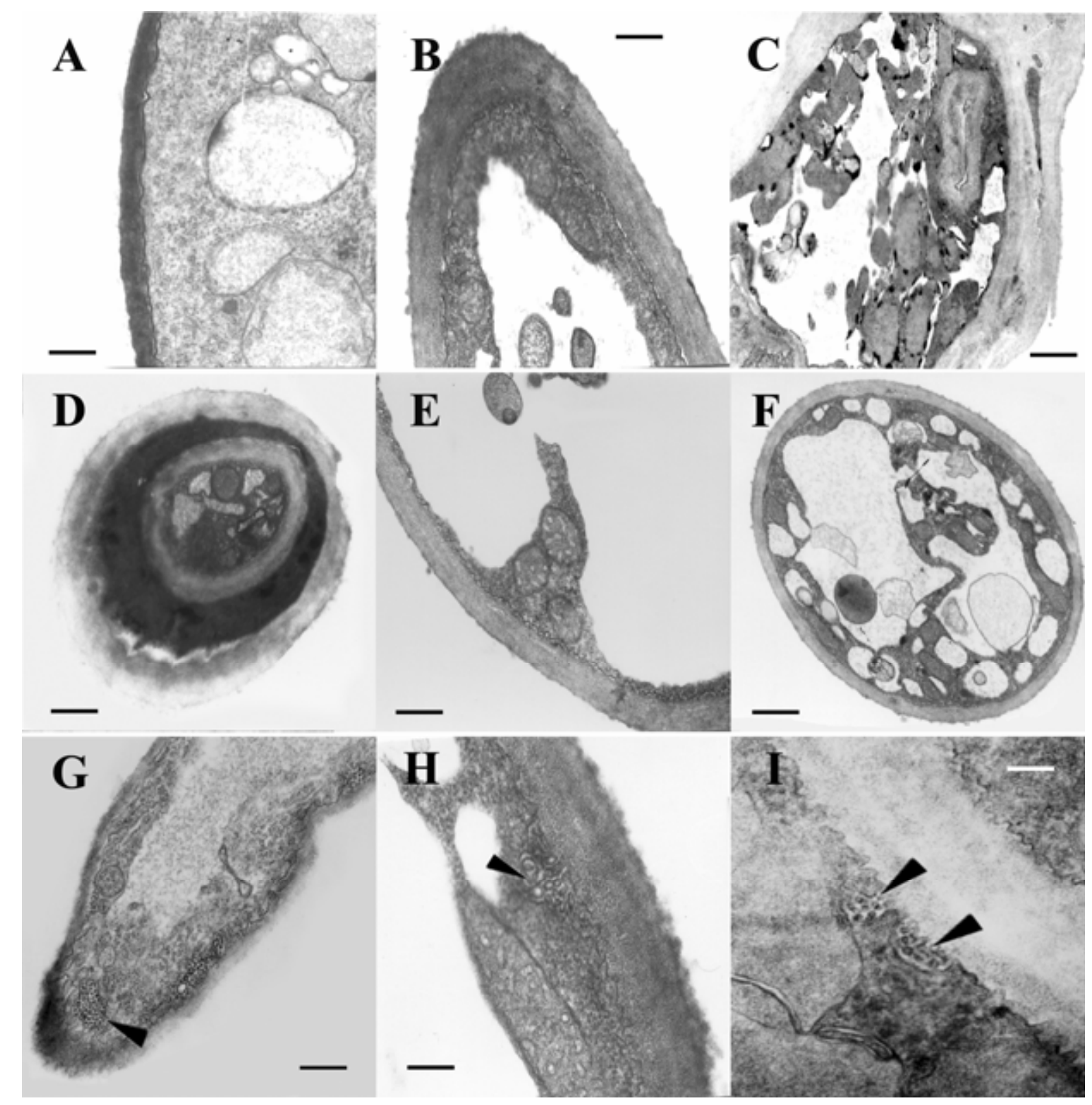

Fig. 6. Flumorph induces abnormal hyphal ultrastructure in Phytophthora melonis. B, Thickened and $\mathbf{C}$ and $\mathbf{D}$, multiple layers of cell walls formed after exposure to flumorph at $25.0 \mathrm{mg} /$ liter compared with A, normal cell walls. G, Normal wall, secretory vesicles (arrowhead), vacuoles in normal hyphae. After treatment with flumorph, F, abundant vacuoles in hyphae and accumulations of wall vesicles near $\mathbf{H}$, a thick region of wall (arrowhead) and $\mathbf{I}$, multiple layers (arrowhead). However, E, mitochondria and F, nuclei appeared normal. Bars = $0.4 \mu \mathrm{m}(\mathbf{H}$ and $\mathbf{I}), 1.2 \mu \mathrm{m}(\mathbf{A}, \mathbf{B}, \mathbf{C}$, and E), $1.4 \mu \mathrm{m}(\mathbf{G}), 1.5 \mu \mathrm{m}(\mathbf{D})$, and $1.7 \mu \mathrm{m}(\mathbf{F})$. 
that in normal hyphae $(P<0.01)$. However, a characteristic feature of hyphae of sensitive isolates exposed to flumorph was the periodic swelling along hyphae, and each expansive region was associated with a nucleus (Fig. 7C). These were most prevalent in the regions of hyphae that underwent swelling.

\section{DISCUSSION}

Flumorph did not inhibit the synthesis of cell walls but disrupted the polar growth of $P$. melonis. The results of calcofluor white staining suggested that, whether treated with flumorph or not, zoospores of $P$. melonis could switch into cystospores accompanied with the formation of cell wall. This suggests that flumorph cannot inhibit the synthesis of cell wall materials, which is consistent with our observations by radioactive tracing (data not shown). However, the deposition of newly synthesized cell wall materials during cystospore germination was disturbed by flumorph. Commonly, a spore is initiated by an isotropic growth phase generating spherical germ cells. The spore then switches from an isotropic to a polar growth, with the uniform distribution of cellulose throughout the cell wall converging to a central point where the hyphal tube is formed $(5,30)$. After treatment with flumorph, however, cystospores failed to induce the isotropic-polar growth and, thus, did not produce germ tubes. Interestingly, they can continue the isotropic growth phase, which is marked by a uniform distribution of newly synthesized cell wall materials throughout the cell. In hyphae, the tip growth was disrupted and the periodic swellings along hyphae were formed after exposure to flumorph (Fig. 3). These morphological changes of hyphae coincided with the effect of dimethomorph on the hyphae of $P$. infestans (19). The polar deposition pattern of newly synthesized cell walls in hyphae also was disrupted after exposure to flumorph. Cellulose and other cell wall materials were deposited uniformly throughout the diffuse expanded cell walls, in contrast to their normal polarized patterns of deposition. When flumorph was washed out, the polarized deposition patterns of cell wall materials were reversed to normal development. These results suggested that flumorph did not inhibit the synthesis of cell wall materials but interfered with the polarized distribution of newly synthesized cell walls. These data also indirectly proved a previous hypothesis that dimethomorph, the analog of flumorph, interfered with the assembly of wall polymers in the fungal cell (19).

Flumorph disrupted the polar deposition of cell wall materials through impairing the F-actin organization. FITCphalloidin staining showed that flumorph disrupted the organization of F-actin. The loss of polar deposition of cell wall materials and the subapical periodic swelling during cystospore germination and hyphal growth probably were mediated by F-actin
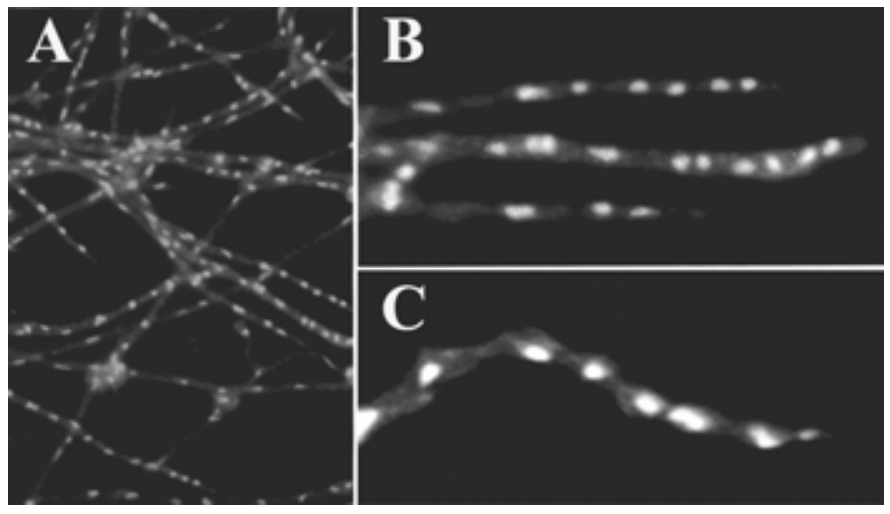

Fig. 7. Effect of flumorph on nuclear distribution in hyphae of Phytophthora melonis. A, Distribution of nuclei in normal hyphae; $\mathbf{B}$ and $\mathbf{C}$, nuclei distribution in the hyphae of resistant isolate FR-58 and wild-type isolate TJ58 , respectively, after treatment with flumorph at $25 \mathrm{mg} / \mathrm{liter}$. disruption because these phenotypes resembled those induced by cytochalasins (28) and latrunculin A and B $(3,4)$, F-actin inhibitors with a different binding site $(3,4,7)$. In addition, earlier studies suggested that secretion of the cell wall components and other cell wall substances and regulating factors is a polarizing process (18). F-actin appears to be an important regulatory component in this polar process $(11,15,16)$. Commonly, vesicles carrying cell-wall-remodeling enzymes and new cell wall constituents probably are secreted by Golgi bodies. Then the actin cytoskeleton directs the delivery of post-Golgi secretory vesicles for the apical growth of hyphae $(12,13,23,25)$. Once the F-actin was disrupted, apical growth of hyphae was lost and abundant subapical expansion occurred, the polar form of which was increasingly lost with increasing actin disruption, culminating in diffuse subapical expansion $(3,4,23)$.

Flumorph disrupted the cytoplasmic organization. The disruption of F-actin, imposed by flumorph, was accompanied by disorganized cytoplasm. After exposure to flumorph, wall vesicles no longer secreted to the apical region of hyphae but adjacent to the subapical cell wall. This resulted in the aberrant deposition of newly synthesized cell wall materials and hyphal periodic expansion. Altered deposition patterns of cell walls also were manifested as thickening and in the development of more than one cell wall layer (Fig. 6B to D). Flumorph did not disrupt DNA replication and nuclear division, but each swelling of hyphae was associated with a nucleus, which resembled the phenotype of Saprolegnia after treatment with latrunculin B (4). This phenotype also may be the result of disruption of F-actin. After F-actin was disrupted, the vesicles did not polarize secretion to the apical of hyphae but diffused around nuclei for subapical growth; thus, each swelling of hyphae was associated with a nucleus $(4,25)$. Upon removing flumorph, the tip growth was recovered and the normal F-actin reorganized. The results of cell wall aberrant deposition also coincided with the effects of dimethomorph on the ultrastructure of $P$. infestans, $P$. erythroseptica, and $P$. cactorum (19). Because of the similar effects of flumorph and dimethomorph on the morphology and ultrastructure of hyphae, we further proved the hypothesis that flumorph and dimethomorph have the same mode of action.

In conclusion, flumorph may penetrate into cells to disrupt the organization of F-actin, resulting in loss of polar deposition of cell wall materials and formation of swellings. However, the way flumorph disrupts the organization of F-actin may be complicated because the assembly of F-actin can be modulated by various factors. The mutations of actin $(9,25)$ or actin-binding protein $(10,23)$ and F-actin inhibitors (4) all can cause the disruption of F-actin and show phenotypes similar to our results. In addition, the mutants of upstream regulators of F-actin, such as Cdc42, which can activate actin cytoskeleton polar orientation $(1,17)$, forming signaling effectors for assembly and polarization of Factin (8), also resulted in loss of the organization of F-actin. All these similar phenotypes were directly or indirectly mediated by F-actin disruption and resulted in the alterations in fungal wall deposition. Thus, it will be important to determine whether flumorph acts on actin or other modulation process.

\section{ACKNOWLEDGMENTS}

This work was supported by the Shenyang Research Institute of Chemical Industry of China and National Science Foundation (30400294). We thank C. N. Mortensen for critical reading of the manuscript.

\section{LITERATURE CITED}

1. Adams, A. E. M., Johnson, D. J., Longnecker, R. M., Sloat, B. F., and Pringle, J. R. 1990. CDC42 and CDC43, two additional genes involved in budding and establishment of cell polarity in the yeast Saccharomyces cerevisiae. J. Cell Biol. 111:131-142.

2. Albert, G., Thomas, A., and Guehne, M. 1991. Fungicide activity of 
dimethomorph on different stages in the life cycle of Phytophthora infestans and Plasmopara viticola. Pages 887-894 in: Association Nationale de Protection des Plantes-3rd Intl. Conf. Plant Dis. Bordeaux, France.

3. Ayscough, K. R., Stryker, J., Pokala, N., Sanders, M., Crews, P., and Drubin, D. G. 1997. High rates of actin filament turnover in budding yeast and roles for actin in establishment and maintenance of cell polarity revealed using the actin inhibitor Latrunculin-A. J. Cell Biol. 137:399416.

4. Bachewich, C., and Heath, I. B. 1998. Radial F-actin arrays precede new hypha formation in Saprolegnia. Implications for establishing polar growth and regulating tip morphogenesis. J. Cell Sci. 111:2005-2016.

5. Bartnicki-Garcia, S., and Lippman, E. 1977. Polarization of cell wall synthesis during spore germination of Mucor rouxii. Exp. Mycol. 1:230-240.

6. Cohen, Y., Baider, A., and Cohen, B. H. 1995. Dimethomorph activity against Oomycete fungal plant pathogens. Phytopathology 85:1500-1506.

7. Cooper, J. A. 1987. Effects of cytochalasin and phalloidin on actin. J. Cell Biol. 105:1473-1478.

8. Evangelistal, M., Zigmond, S., and Boone, C. 2003. Formins: Signaling effectors for assembly and polarization of actin filaments. J. Cell Sci. 116:2603-2611.

9. Gabriel, M., and Kopecka, M. 1995. Disruption of the actin cytoskeleton in budding yeast results in formation of an aberrant cell wall. Microbiology 141:891-899.

10. Goodson, H. V., Anderson, B. L., Warrick, H. M., Pon, L. A., and Spudich, J. A. 1996. Synthetic lethality screen identifies a novel yeast myosin gene (MYO5): Myosin 1 proteins are required for polarization of the actin cytoskeleton. J. Cell Biol. 133:1277-1291.

11. Heath, I. B. 1990. The roles of actin in tip growth of fungi. Int. Rev. Cytol. 123:95-127.

12. Heath, I. B., and Kaminskyj, S. G. W. 1989. The organization of tipgrowth related organelles and microtubules revealed by quantitative analysis of freeze-substituted Oomycete hyphae. J. Cell Sci. 93:41-52.

13. Heath, I. B., Rethoret, K., Arsenault, A. L., and Ottensmeyer, F. P. 1985. Improved preservation of the form and contents of wall vesicles and the Golgi apparatus in freeze-substituted hyphae of Saprolegnia. Protoplasma 128:81-93

14. Herth, W. 1980. Calcofluor white and Congo red inhibit chitin microfibril assembly of Poterioochromonas: Evidence for a gap between polymerization and microfibril formation. J. Cell Biol. 87:442-450.

15. Irazoqui, J. E., and Lew, D. J. 2004. Polarity establishment in yeast. J. Cell Sci. 117:2169-2171.

16. Jackson, S. L., and Heath, I. B. 1990. Evidence that actin reinforces the extensible hyphal apex of the Oomycete Saprolegnia ferax. Protoplasma 157:144-153.

17. Johnson, D. I. 1999. Cdc42: An essential Rho-type GTPase controlling eukaryotic cell polarity. Microbiol. Mol. Biol. Rev. 63:54-105.

18. Kopecká, M., Feet, G. H., and Phaff, H. 1995. Ultrastructure of the cell wall of Schizosaccharomyces pombe following treatment with various glucanases. J. Struct. Biol. 114:140-152.

19. Kuhn, P. J., Pitt, D., and Lee, S. A. 1991. Effects of dimethomorph on the morphology and ultrastructure of Phytophthora. Mycol. Res. 95:333340.

20. Liu, W. C., Li, Z. L., Zhang, Z. J., and Liu, C. L. 2000. Antifungal activity and prospect of flumorph and its mixtures. Zhejiang Chem. Ind. 31:8788.

21. Liu, W. C., and Liu, C. L. 2001. New high efficiency fungicide flumorph. J. Pestic. 41:8-11.

22. Marks, J., Hagan, I. M., and Hyams, J. S. 1986. Growth polarity and cytoskeleton. J. Cell Sci. 5(Suppl.):229-241.

23. McGoldrick, C. A., Gruver, C., and May, G. S. 1995. MyoA of Aspergillus nidulans encodes an essential myosin 1 required for secretion and polarized growth. J. Cell Biol. 128:577-587.

24. Mitani, S., Araki, S., Yamaguchi, T., Takii, Y., Ohshima, T., and Matsuo, N. 2001. Antifungal activity of the novel fungicide cyazofamid against Phytophthora infestans and other plant pathogenic fungi in vitro. Pestic. Biochem. Physiol. 70:92-99.

25. Mulholland, J., Wesp, A., Riezman, H., and Botstein, D. 1997. Yeast actin cytoskeleton mutants accumulate a new class of Golgi-derived secretory vesicle. Mol. Biol. Cell 8:1481-1499.

26. Riemann, M., Biiche, C., Kassemeyer, H. H., and Nick, P. 2002. Cytoskeletal responses during early development of the downy mildew of grapevine (Plasmopara viticola). Protoplasma 219:13-22.

27. Takagi, T., Ishijima, S. A., Ochi, H., and Osumi, M. 2003. Ultrastructure and behavior of actin cytoskeleton during cell wall formation in the fission yeast Schizosaccharomyces pombe. J. Electron Microsc. 52:161174.

28. Torralba, S., Raudaskoski, M., Pedregosa, A. M., and Laborda, F. 1998 Effect of cytochalasin A on apical growth, actin cytoskeleton organization and enzyme secretion in Aspergillus nidulans. Microbiology (UK) 144:45-53.

29. vandenBerg, A. L., Ibrahim, A. S., Edwards, J. E., Toenjes, K. A., and Johnson, D. I. 2004. Cdc42p GTPase regulates the budded-to-hyphalform transition and expression of hypha-specific transcripts in Candida albicans. Eukaryotic Cell 3:724-734.

30. Wendland, J., and Philippsen, P. 2001. Cell polarity and hyphal morphogenesis are controlled by multiple Rho-protein modules in the filamentous ascomycete Ashbya gossypii. Genetics 157:601-610.

31. Yuan, S. K., Liu, X. L., Si, N. G., Dong, J., Gu, B. G., and Jiang, H. 2006. Sensitivity of Phytophthora infestans to flumorph: In vitro determination of baseline sensitivity and the risk of resistance. Plant Pathol. 55:258-263.

32. Zhu, S. S., Liu, X. L., Li, J. Q., and Si, N. G. 2006. Uptake and translocation behavior of new fungicide flumorph in cucumber plant. Chem. J. Chin. Univ. 27:1887-1890.

33. Zhu, S. S., Yuan, S. K., Fan, J. R., Wang, Y., Liu, M., and Liu, X. L. 2005. The effect of six fungicides on different developmental stages in the life cycle of Pseudoperonospora cubensis. Chin. J. Pestic. Sci. 7:119-125. 